Supporting Information for

\title{
Dynamic-then-Static Approach for Core Excitations of Open-Shell Molecules
}

\author{
Ruoqi Zhao, ${ }^{1,2}$ Adam Grofe, ${ }^{2}$ Zikuan Wang, ${ }^{3}$ Peng Bao, ${ }^{4}$ Xin Chen, ${ }^{*, 1}$ \\ Wenjian Liu, $*, 3$ and Jiali Gao*,1,5
}

1. Institute of Systems and Physical Biology,

Shenzhen Bay Laboratory, Shenzhen, Guangdong 518055, China

2. Institute of Theoretical Chemistry, Jilin University

Changchun, Jilin 130023, China

3. Qingdao Institute for Theoretical and Computational Sciences, Institute of Frontier and Interdisciplinary Science, Shandong University, Qingdao, Shandong 266237, China

4. Beijing National Laboratory for Molecular Sciences, State Key Laboratory for Structural Chemistry of Unstable and Stable Species, Institute of Chemistry, Chinese Academy of Sciences, Beijing 100190, China

5. Department of Chemistry and Supercomputing Institute, University of Minnesota, Minneapolis, Minnesota 55455, United States

This supporting information (SI) contains the optimized geometries for the open-shell species discussed in the text and a schematic illustration of the relationship between determinant energy and exchange integrals given in (Eqs 3 and 5). 
Table S1. Optimized geometries using U-M06-2X/cc-pQVZ.

1. $\mathrm{N}_{2}{ }^{+}, \mathrm{S}=1 / 2$

$\begin{array}{lllll}1 & 7 & 0.000000 & 0.000000 & 0.548961\end{array}$

$\begin{array}{llllll}2 & 7 & 0.000000 & 0.000000 & -0.548961\end{array}$

2. $\mathrm{NO}, \mathrm{S}=1 / 2$

$\begin{array}{lllll}1 & 7 & 0.000000 & 0.000000 & -0.605704\end{array}$

$\begin{array}{llllll}2 & 8 & 0.000000 & 0.000000 & 0.529991\end{array}$

3. $\mathrm{NO}_{2}, \mathrm{~S}=1 / 2$

$\begin{array}{lllll}1 & 8 & 0.000000 & 1.088832 & -0.137193\end{array}$

$\begin{array}{lllll}2 & 7 & 0.000000 & 0.000000 & 0.313584\end{array}$

$\begin{array}{llllll}3 & 8 & 0.000000 & -1.088832 & -0.137193\end{array}$

4. $\mathrm{NH}^{+}, \mathrm{S}=3 / 2$

$\begin{array}{lllll}1 & 7 & 0.000000 & 0.000000 & 0.137857\end{array}$

$\begin{array}{llllll}2 & 1 & 0.000000 & 0.000000 & -0.964998\end{array}$

5. $\mathrm{NH}^{+}, \mathrm{S}=1 / 2$

$\begin{array}{lllll}17 & 0.000000 & 0.000000 & 0.134076\end{array}$

$\begin{array}{lllll}2 & 1 & 0.000000 & 0.000000 & -0.938531\end{array}$

6. $\mathrm{NH}_{2}^{+}, \mathrm{S}=1$

$\begin{array}{llllll}1 & 7 & 0.000000 & 0.000000 & 0.056018\end{array}$

$\begin{array}{llllll}2 & 1 & 0.000000 & 1.000559 & -0.196063\end{array}$

$\begin{array}{llllll}3 & 1 & 0.000000 & -1.000559 & -0.196063\end{array}$

7. $\mathrm{C}_{6} \mathrm{H}_{6}{ }^{+}, \mathrm{S}=1 / 2$

$\begin{array}{llllll}1 & 6 & 0.720104 & -1.182291 & -0.000000\end{array}$

$\begin{array}{llllll}2 & 6 & 1.428781 & -0.000389 & -0.000000\end{array}$

$\begin{array}{llllll}3 & 6 & 0.720855 & 1.181878 & 0.000000\end{array}$

$\begin{array}{llllll}4 & 6 & -0.720129 & 1.182288 & -0.000000\end{array}$

$\begin{array}{llllll}5 & 6 & -1.428781 & 0.000398 & -0.000000\end{array}$

$\begin{array}{lllll}6 & 6 & -0.720830 & -1.181881 & 0.000000\end{array}$

$\begin{array}{llllll}7 & 1 & 1.231029 & -2.125780 & -0.000000\end{array}$

$\begin{array}{llllll}8 & 1 & 2.499513 & -0.000748 & -0.000000\end{array}$

$\begin{array}{llllll}9 & 1 & 1.232282 & 2.125094 & 0.000000\end{array}$

$\begin{array}{llllll}10 & 1 & -1.231024 & 2.125792 & -0.000000\end{array}$

$\begin{array}{llllll}11 & 1 & -2.499514 & 0.000707 & -0.000000\end{array}$

$\begin{array}{llllll}12 & 1 & -1.232287 & -2.125081 & 0.000000\end{array}$

8. $\mathrm{CH}_{3}, \mathrm{~S}=1 / 2$ 

$\begin{array}{lllll}1 & 6 & 0.000000 & 0.000000 & 0.00000\end{array}$
$\begin{array}{llllll}2 & 1 & 0.000000 & 1.075552 & 0.00000\end{array}$
$\begin{array}{llllll}3 & 1 & 0.931455 & -0.537776 & 0.00000\end{array}$
$\begin{array}{llllll}4 & 1 & -0.931455 & -0.537776 & 0.00000\end{array}$
9. $\mathrm{CO}^{+}, \mathrm{S}=1 / 2$

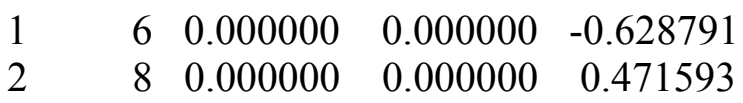

10. $\mathrm{NH}_{2}, \mathrm{~S}=1 / 2$

$\begin{array}{lllll}1 & 7 & -0.000000 & -0.000000 & 0.141122\end{array}$

$\begin{array}{llllll}2 & 1 & 0.000000 & 0.803032 & -0.493928\end{array}$

$\begin{array}{llllll}3 & 1 & -0.000000 & -0.803032 & -0.493928\end{array}$

11. $\mathrm{NH}_{3}{ }^{+}, \mathrm{S}=1 / 2$

$\begin{array}{lllll}1 & 7 & -0.000000 & 0.000000 & -0.000026\end{array}$

$\begin{array}{lllll}2 & 1 & 0.000000 & 1.022998 & 0.000060\end{array}$

$\begin{array}{lllll}3 & 1 & -0.885943 & -0.511499 & 0.000060\end{array}$

$\begin{array}{lllll}4 & 1 & 0.885943 & -0.511499 & 0.000060\end{array}$

12. $\mathrm{OH}, \mathrm{S}=1 / 2$

$\begin{array}{lllll}1 & 8 & 0.000000 & 0.000000 & 0.107836\end{array}$

$\begin{array}{llllll}2 & 1 & 0.000000 & 0.000000 & -0.862687\end{array}$

13. $\mathrm{OOH}, \mathrm{S}=1 / 2$

$\begin{array}{lllll}1 & 8 & 0.054937 & 0.706511 & 0.000000\end{array}$

$\begin{array}{llllll}2 & 8 & 0.054937 & -0.598476 & 0.000000\end{array}$

$\begin{array}{lllll}3 & 1 & -0.878993 & -0.864277 & 0.000000\end{array}$ 
Figure S1. Schematic definition of the determinants and orbital exchange relationship for $\mathrm{NH}_{2}{ }^{+}$ in the triplet spin $(S=1)$ manifold, with respect to the high-spin configuration $(S=2)$.

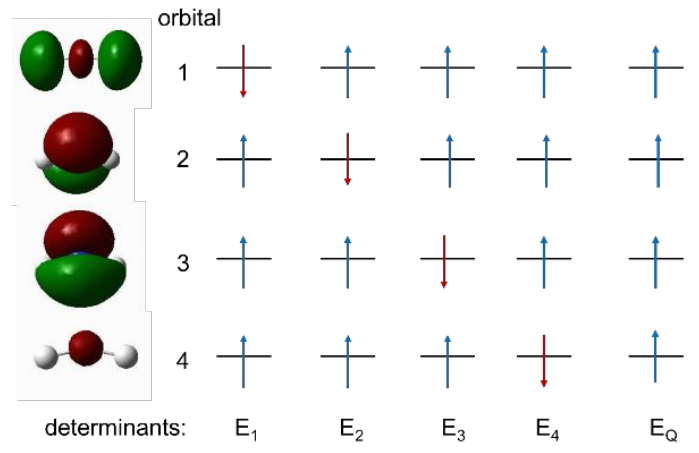

Define the energy difference matrix: $\Delta \mathbf{E}_{B L K S}=\left(\begin{array}{c}E_{1}-E_{Q} \\ E_{2}-E_{Q} \\ E_{3}-E_{Q} \\ E_{4}-E_{Q}\end{array}\right)$

Define the matrix for the exchange in order: $K_{M S D F T}=\left(\begin{array}{l}K_{12} \\ K_{13} \\ K_{14} \\ K_{23} \\ K_{24} \\ K_{34}\end{array}\right)$

Then, the constraint for the $M_{S}=1$ projection of the quintet state, $\left|S, M_{S}>=\right| 2,1>$ multiplet is

$$
\Delta \mathbf{E}_{B L K S}=-\mathbf{M}^{T} \mathbf{K}_{M S D F T}
$$

where the matrix $\mathbf{M}$ is given by

$$
\mathbf{M}=\left(\begin{array}{llll}
1 & 1 & 0 & 0 \\
1 & 0 & 1 & 0 \\
1 & 0 & 0 & 1 \\
0 & 1 & 1 & 0 \\
0 & 1 & 0 & 1 \\
0 & 0 & 1 & 1
\end{array}\right)
$$

